\title{
ORDERED SEMIGROUPS
}

\author{
PAUL CONRAD ${ }^{1)}$
}

1. Introduction. In this paper order will always mean linear or total order, and, unless otherwise stated, the composition of any semigroup will be denoted by + . A semigroup $S$ is an ordered semigroup (notation o.s.) if $S$ is an ordered set and for all $a, b, c$ in $S$

$$
a<b \text { implies } a+c<b+c \text { and } c+a<c+b .
$$

If in addition $a+a>a$ for all $a$ in $S$, then we call $S$ a positvve ordered semigroup (notation pos. o.s.). In particular an o.s. $S$ is cancellative, and hence if $e$ is an idempotent element of $S$, then $e$ is the identity for $S$. Moreover, for $a$. $b, c$ in $S$ and $n$ a positive integer we have the following rules

$$
\begin{aligned}
& a>b \leftrightarrow a+c>b+c \leftrightarrow c+a>c+b . \\
& a>b \leftrightarrow n a>n b . \\
& a>b \text { and } c>d \rightarrow a+c>b+d .
\end{aligned}
$$

Let $\Gamma$ be an ordered set, and for each $\gamma \in \Gamma$ let $S_{\gamma}$ be an o.s. such that $S_{\alpha} \cap S_{\beta}=\square$ (the null set) if $\alpha \neq \beta$. Consider $a \in S_{\alpha}$ and $b \in S_{\beta}$ where $\alpha \leq \beta$. Define $a<b$ if $\alpha<\beta$ or $\alpha=\beta$ and $a<b$ in $S_{\alpha}$. Define $a+b=b+a=b$ if $\alpha<\beta$ and use the addition in $S_{\alpha}$ if $\alpha=\beta$. Then $Q=\bigcup_{\gamma \in \Gamma} S_{\curlyvee}$ is an ordered set and a semigroup-the ordinal sum of the $S_{r}$. The $S_{r}$ are the components of $Q$.

In section 3 we give a necessary and sufficient condition for a semigroup $S$ to be the ordinal sum of pos. o.s. (Theorem 3-1). We also show that if $S$ is a pos. o.s., then there exists a rather natural $o$-homomorphism of $S$ onto an ordinal sum of pos. o.s. each of which is $o$-isomorphic to a semigroup of positive real numbers. Cheheta [2] and Vinogradov [9] use an example of Malcev to show that an o.s. cannot necessarily be embedded in a group. Ore [8] has shown that if every pair of elements in a semigroup $S$ has a common right multiple, then $S$ can be embedded in a group $G=\{a-b: a, b \in S\} . G$ is called

1) This work was supported by a grant from the National Science Foundation. 
the difference group of $S$. We show that if $S$ is an o.s., then the order of $S$ can oe extended to an order of $G$ in one and only one way. In section 5 we show that the order type of the set of all convex normal subgroups of $G$ is determined by $S$.

2. Embedding theorems. Throughout this section $S$ will denote an o.s.

Theorem 2-1. Suppose that S satisfies: (*) for each pair $a, b$ in $S$ there exists a pair $x, y$ in $S$ such that $a+x=b+y$. Then there exists an o-group $G$ such that $G=\{a-b: a, b \in S\}$ and $a-b$ is positive in $G$ if and only if $a>b$ in $S$. Moreover, if $H$ is an o-group that contains $S$ as an ordered subsemigroup and is generated by $S$, then there exists an o-isomorphism $\pi$ of $G$ onto $H$ such that $s \pi=s$ for all $s \in S$. We call $G$ the difference group of $S$.

This theorem is a corollary of a result of Ore [8] for integral domains. We outline the construction of an $o$-group $G^{\prime}$ that is $o$-isomorphic to $G$. Let $T=S \times S$ and define that $(a, b) \sim(c, d)$ if there exist $x, y$ in $S$ such that $a+x$ $=c+y$ and $b+x=d+y$. Then $\sim$ is an equivalence relation. Denote the equivalence class containing $(a, b)$ by $[a, b]$, and define that $[a, b]+[c, d]$ $=[a+x, d+y]$ where $b+x=c+y$. Then the set $G^{\prime}$ of all equivalence classes is a group, $[a, a]$ is the identity, $[b, a]$ is the inverse of $[a, b]$, and the mapping $\tau$ of $s$ upon $[s+x, x]$ is an isomorphism of $S$ into $G^{\prime}$.

$[a, b]=[a+x, x]-[b+x, x]=a \tau-b \tau$. Thus there is at most one way of extending the order of $S$ to an ordering of $G^{\prime}$. Namely, define that $[a, b]$ is positive in $G^{\prime}$ if $a>b$ in $S$. Let $\mathscr{P}$ be the set of all positive elements in $G^{\prime}$. If $[a, a] \neq[b, c]$, then $b>c$ or $b<c$ in $S$, and hence $[b, c] \in \mathscr{P}$ or $-[b, c]$ $=[c, b] \in \mathscr{P}$. If $[a . b]$ and $[c, d]$ belong to $\mathscr{P}$, then $a>b$ and $c>d$, and $[a, b]+[c, d]=[a+x, d+y]$ where $b+x=c+y$. Thus $a+x>b+x=c+y$ $>d+y$, and hence $[a, b]+[c, d] \in \mathscr{P}$. If $[a, b] \in \mathscr{P}$ and $[c, d] \in G^{\prime}$, then $X=[d, c]+[a, b]+[c, d]=[d, c]+[a+x, d+y]=[d+u, d+y+v]$ where $b+x=c+y$ and $c+u=a+x+v$. To show that $X \in \mathscr{P}$ it suffices to show that $u>y+v$. Pick $r$ and $s$ in $S$ such that $u+r=y+s$. Then $a+x+v+r=c+u+r$ $=c+y+s=b+x+s$. If $v+r \geq s$, then $a+x+v+r>b+x+s$ because $a>b$. Thus $v+r<s$, and hence $y+v+r<y+s=u+r$. Therefore $y+v<u$.

Finally suppose that $H$ is an $o$-group that is generated by $S$. Let $[a, b] \pi^{\prime}$ $=a-b$ for all $[a, b] \in G^{\prime}$. If $[a, b]=[c, d]$, then $a+x=c+y$ and $b+x=d+y$. 
Thus $a-b=a+x-x-b=c+y-y-a=c-d$, and hence $\pi^{\prime}$ is single valued. $([a, b]+[c, d]) \pi^{\prime}=[a+x, d+y] \pi^{\prime}=a+x-y-d$, where $b+x=c+y$. Thus $x-y=-b+c$ and $a+x-y-d=a-b+c-d=[a, b] \pi^{\prime}+[c, d] \pi^{\prime}$. If $0=[a, b] \pi^{\prime}$ $=a-b$, then $[a, b]$ is the identity of $G^{\prime}$. If $[a, b] \in \mathscr{P}$, then $a>b$ in $S$ and hence in $H$. Thus $[a, b] \pi^{\prime}=a-b$ is positive in $H . \quad S \leq G^{\prime} \pi^{\prime} \leq H$ and, since $H$ is generated by $S, G^{\prime} \pi^{\prime}=H$. Therefore $\pi^{\prime}$ is an $o$-isomorphism of $G^{\prime}$ onto $H$. This completes the proof of the theorem.

Corollary I. S satisfies $(*)$ if and only if $S$ can be embedded in an ogroup $G=\{a-b: a, b \in S\}$.

For suppose that $G=\{a-b: a, b \in S\}$ and that $a$ and $b$ belong to $S$. Then $-a+b \in G$ and hence $-a+b=x-y$ for some $x, y \in S$. Thus $b+y=a+x$.

Corollary II. Suppose that $S$ satisfies $(*)$ and let $G$ be the difference group of $S$. Then for $a, b, c$ in $S$

(a) $a-b=c-d$ if and only if there exist $x, y$ in $S$ such that $a+x=c+y$ and $b+x=d+y$.

(b) $a-b+c-d=a+x-(d+y)$ for all $x, y$ in $S$ such that $b+x=c+y$.

(c) $a-b>c-d$ if and only if there exist $x, y$ in $S$ such that $a+x>c+y$ and $b+x=d+y$.

The equivalence of (i) and (ii) in the following corollary is well known and has been proven by Tamari, Alimov, and Nakada ([4] p. 309).

COROLlARY III. For a commutative semigroup $A$ the following are equivalent.

( i ) A can be embedded in an o-group.

(ii) A can be ordered.

(iii) A satisfies the cancellation law, and $n a=n b$ implies that $a=b$, for all $a, b$ in $A$ and all positive integers $n$.

Proof. Clearly (i) implies (ii), and since any commutative o.s. satisfies (*), (ii) implies (i). An easy argument shows that (ii) implies (iii). Finally assume that $A$ is cancellative, and let $G=\{a-b: a, b \in A\}$ be the difference group of $A$. If $x=a-b \in G$ and $n x=0$, then $0=n x=n a-n b$, and hence $n a$ $=n b$. Thus by (iii) $a=b$, and $0=a-b=x$. Therefore (iii) implies that the difference group $G$ of $A$ exists and is abelian and torsion free. But this means that $G$ can be ordered (see for example [7]). 
Suppose that $A$ is a cancellative commutative semigroup with identity 0 . Then if $A$ can be ordered, it is torsion free, but the converse is false. For consider the semigroup $B=N \oplus N$, where $N$ is the additive semigroup of nonnegative integers. For $(a, b)$ and $(c, d)$ in $B$ define that $(a, b) \sim(c, d)$ if $a \equiv c \bmod 2, b \equiv d \bmod 2$ and $a+b=c+d$. Then it is easy to show that $\sim$ is a congruence relation. Let $[a, b]$ be the congruence class that contains $(a, b)$. $B / \sim=\{[a, b]: a, b \in N\}=\{[2 n, 0],[2 n+1,0],[0,2 n+1]$ and $[2 n+1,1]$ for all $n \in N\}$ is a commutative semigroup with identity [0,0]. It is easy to show that $B / \sim$ satisfies the cancellation law and is torsion free, but $2[1,1]$ $=2[0,2]$ and $[1,1] \neq[0,2]$. Thus (iii) of the last corollary is not satisfied, and hence $B / \sim$ cannot be ordered.

Let $P=\{x \in S: x+x>x\}$ and $N=\{x \in S: x+x<x\}$. The following five propositions are easy to verify (or see [1] for proofs).

1) $P=\{x \in S: x+s>s$ for all $s \in S\}=\{x \in S: s+x>s$ for all $s \in S\}$.

2) $N=\{x \in S: x+s<s$ for all $s \in S\}=\{x \in S: s+x<s$ for all $s \in S\}$.

3) $P$ and $N$ are subsemigroups of $S$.

4) $N<P$. That is, $n<p$ for all $n \in N$ and all $p \in P$.

5) If $S$ does not have an identity, then $S=N \cup P$ and an identity 0 can be adjoined to $S$ so that $T=S \cup\{0\}$ is a semigroup. Moreover, the order of $S$ can be extended to an order of $T$ in one and only one way, namely $N<0<P$. If we adjoin an identity to a pos. o.s we shall call the result a pos. o.s. with zero. An o.s. $S$ is naturally ordered if for all $a, b$ in $S$

( R) $a>b$ implies $a=b+x$ for some $x$ in $S$, and

(L) $a>b$ implies $a=x+b$ for some $x$ in $S$.

Note that a pos. o.s. $P$ satisfies $(R)$ if and only if $b+P=\{a \in P: a>b\}$ for all $b$ in $P$.

Theorem 2-2. If $S$ satisfies $(R)$, then $S$ satisfies $(*)$ and hence $S$ is an ordered subsemigroup of its difference group $G$. If $S$ is naturally ordered, then $S$ contains the semigroup of all positive elements of $G$. $A$ pos. o.s. $P$ is the semigroup of all positive elements of an o-group if and only if $P$ is naturally ordered.

Proof. Consider $a, b$ in $S$. If $a>b$, then $a=b+x$ for some $x$ in $S$. Thus $a+b=b+(x+b)$. Similarly if $a \leq b$, then $a+u=b+v$ for some $u, v$ in $S$. Therefore $S$ satisfies $(*)$. Suppose that $S$ is naturally ordered, and consider $a^{*}$ 
positive element $y$ in the difference group $G$ of $S$. $y=a-b$, where $a, b \in S$ and $a>b$. Thus $a=x+b$ for some $x \in S$, and hence $y=a-b=x \in S$.

Finally suppose that $P$ is a naturally pos. o.s. and let $\mathscr{P}$ be the semigroup of all positive elements of the difference group $G$ of $P$. Then we have shown that $P \supseteq \mathscr{P}$. If $p \in P$, then $p+p>p$ in $P$ and hence $p=p+p-p>0$ in $G$. Therefore $P \subseteq \mathscr{g}$.

Lemma 2-1. Let $a, b, c$ be elements of $S$. If $a+b<b+a$, then $a+n b$ $<n b+a$ and $n a+n b \leq n(a+b)<n(b+a) \leq n b+n a$ for all positive integers $n$, where the equalities hold if and only if $n=1$.

This follows by a simple induction argument or see [6] for a proof.

Corollary. If $p$ and $q$ are positive integers and $p a=q b$, then $a+b=b+a$.

For if $a+b<b+a$, then $(p+1) a=a+p a=a+q b<q b+a=p a+a$ $=(p+1) a$, a contradiction.

Note that Lemma 2-1 and its corollary are true for an ordinal sum of o.s. For if $a+b<b+a$, then $a$ and $b$ belong to the same component. In [6] the following theorem (which we use later) is proven.

THEOREM 2-3. For an o.s. S the following are equivalent. (i) There exists an o-isomorphism of $S$ into a subserigroup of the (naturally ordered) additive group $R$ of real numbers. (ii) For each pair $a<b$ in $S$, there exist positive integers $m$ and $n$ such that $m a<(m+1) b$ and $(n+1) a<n b$.

THEOREM 2-4. Suppose that the center $Z=\{z \in S: z+s=s+z$ for all $s \in S\}$ of $S$ is not empty. Then there exists o.s. $T$ such that

1) $S$ is an ordered subsemigroup of $T$,

2) $T$ contains the difference group $G$ of $Z$ and $T$ is generated by $S$ and $G$,

3) If $T^{\prime}$ is an o.s. that satisfies 1) and 2), then there exists a unique $o$ isomorphism $\pi$ of $T$ onto $T^{\prime}$ such that $s \pi=s$ for all $s \in S$.

We outline a proof, leaving out the straightforward computations. Let $Q=S \times Z$ and for $(a, b)$ and $(c, d)$ in $Q$ define that

$$
\begin{aligned}
& (a, b)+(c, d)=(a+c, b+d) \text { and } \\
& (a, b) \sim(c, d) \text { if } a+d=b+c .
\end{aligned}
$$

Then $Q$ is a semigroup, and $\sim$ is a congruence relation. As usual, denote the equivalence class containing $(a, b)$ by $[a, b]$. For $[a, b]$ and $[c, d]$ in $Q / \sim$ 
define that $[a, b]>[c, d]$ if $a+d>b+c$. Then $(Q / \sim,+,>)$ is an o.s. and the mapping $\tau$ of $a \in S$ upon $[a+z, z]$, where $z$ is a fixed element in $Z$ is an $o$-isomorphism of $S$ into $Q / \sim G^{\prime}=\{[a, b]: a, b \in Z\}$ is the center of $Q / \sim$ and the difference group of $Z \tau$. Clearly $Q / \sim$ is generated $S \tau$ and $G^{\prime}$. Thus there exists an $o$-semigroup $T$ that satisfies 1 ) and 2). Moreover $G$ is the center of $T$. Finally suppose that $T$ and $T^{\prime}$ are o.s. that satisfy 1) and 2), and consider $t \in T . \quad t=s+g=s+z_{1}-z_{2}$, where $s \in S, g \in G$ and $z_{1}, z_{2} \in Z$. Define $t \sigma=\left[s+z_{1}, z_{2}\right]$. Then $\sigma$ is on $o$-isomorphism of $T$ onto $Q / \sim$. Similarly we define an $o$-isomorphism $\sigma^{\prime}$ of $T^{\prime}$ onto $Q / \sim$, and then $\pi=\sigma \sigma^{\prime-1}$ is the desired $o$-isomorphism of $T$ onto $T^{\prime}$.

\section{Positive ordered semigroups}

TheOREm 3-1. A semigroup $S$ is an ordinal sum of pos. o.s. if and only if

( I ) $S$ is an ordered set, and for all $a, b, c$ in $S$,

(II) if $a<b$, then $a+c \leq b+c$ and $c+a \leq c+b$,

(III) $a+a>a$,

(IV) if $a+b=a+c$, then $b=c$ or $a+b=a$, and if $b+a=c+a$, then $b=c$ or $b+a=a$.

Proof. It is easy to verify that an ordinal sum of pos. o.s. satisfies these four conditions. Conversely assume that $S$ is a semigroup that satisfies (I), (II), (III) and (IV). Then $S$ satisfies (III') $a+b \geq \max \{a, b\} \leq b+a$ for all $a$, $b$ in $S$. For if $a+b<a$, then $a+2 b \leq a+b$. If $a+2 b<a+b$, then $2 b<b$, but this contradicts (III). If $a+2 b=a+b$, then by (IV) $2 b=b$ or $a+b=a$, a contradiction. Therefore $a+b \geq a$, and by a similar argument $a+b \geq b$.

For $a, b$ in $S$ we define that $a \sim b$ if $a+b>\max \{a, b\}<b+a$. Clearly $\sim$ is symmetric, and by (III) it is reflexive. Suppose that $a \sim b$ and $b \sim c$. Then $c+b>b$, and thus $a+c+b \geq a+b$. If $a+c+b=a+b$, then by (IV) $c+b=b$ or $a+b=a$. Then $b+c$ or $a+b$, a contradiction. Thus $a+c+b$ $>a+b$, and hence $a+c>a$. By symmetry it follows that $a \sim c$, and hence $\sim$ is an equivalence relation.

Let $\bar{a}=\{b \in A: b \sim a\}$, and consider $b, c$ in $\bar{a}$. We show that $a+b+c$ $>\max \{a, b+c\}$. By symmetry it follows that $b+c \in \bar{a}$, and hence that $\bar{a}$ is a semigroup. If $a+b+c<a+b$, then $b+c<b$, and hence $b+c$. Thus $a+b+c$ $\geq a+b>a$. If $a+b+c<b+c$, then $a+b<b$, and hence $a+b$. If $a+b+c$ 
$=b+c$, then by (IV) $a+b=b$ or $b+c=c$, and hence $a+b$ or $b+c$. Therefore $a+b+c>b+c$.

We next show that $\bar{a}$ is a pos. o.s. Consider $x, y, z$ in $\bar{a}$. If $x<y$, then $x+z<y+z$. For otherwise $x+z=y+z$, and thus $x=y$ or $x+z=z$, a contradiction. By symmetry if $x<y$, then $z+x<z+y$. Thus $\bar{a}$ is an o.s., and since $\bar{a}$ satisfies (III) it is a pos. o.s.

In order to prove that $S$ is the ordinal sum of the semigroups $\bar{a}$ it suffices to show that if $a<b$ and $\bar{a} \neq \bar{b}$, then $a+b=b$ and $\bar{a}<\bar{b} . \quad a+b \leq b$ because $\bar{a} \neq \bar{b}$, and by (III') $a+b \geq b$. Pick $a^{\prime} \in \bar{a}$ and $b^{\prime} \in \bar{b} . a^{\prime}+a+b=a^{\prime}+b$. Hence by (IV) $a^{\prime}+a=a^{\prime}$ or $a^{\prime}+b=b$. But $a^{\prime}+a>a$ because $a^{\prime} \sim a$. If $b^{\prime} \leq a^{\prime}$, then $b^{\prime}+b \leq a^{\prime}+b=b$, and hence $b^{\prime}+b$. Therefore $a^{\prime}<b^{\prime}$, and hence $\bar{a}<\bar{b}$.

For the rest of this section we investigate pos. o.s. The information obtained will then apply to semigroups that satisfy the four properties of Theorem 3-1. For the remainder of this section let $P$ denote $a$ pos. o.s.

Lemma 3-1. For all $a, b$ in $P$ and all positive integers $m,(m+1) a$ $+(m+1) b$ is greater than $m a+m b$ and $m b+m a$.

Proof. $\quad(m+1) a>m a$ and $(m+1) b>m b$. Thus $(m+1) a+(m+1) b$ $>m a+m b$. Suppose that $a \geq b$. If $a \geq m b$, then $(m+1) a+(m+1) b>(m+1) a$ $=a+m a \geq m b+m a$. If $a<m b$, then since $m b<(m+1) b \leq(m+1) a$, there exists a positive integer $n$ such that $n a<(m+1) b \leq(n+1) a$. Thus $(m+1) a$ $+(m+1) b>(m+1) a+n a=(n+1) a+m a>m b+m a$. By an entirely similar argument if $a<b$, then $(m+1) a+(m+1) b>m b+m a$.

Lemma 3-2. For all $a, b$ in $P$ and all positive integers $m$ :

(i ) $(\mathrm{m}+1)(a+b)$ is greater than $m(a+b)$ and $m(b+a)$.

(ii) $(m+1) a+(m+1) b$ is greater than $m(a+b)$ and $m(b+a)$.

(iii) $(m+1)(a+b)$ is greater than $m b+m a$ and $m a+m b$.

Proof. (i) $(m+1)(a+b)=m(a+b)+a+b>m(a+b)$ and $(m+1)(a+b)$ $=a+m(b+a)+b>m(b+a)+b>m(b+a)$. (ii) If $a+b \geq b+a$, then by Lemma $2-1, \quad(m+1) a+(m+1) b \geq(m+1)(a+b)$, and by (i) $(m+1)(a+b)$ $>m(a+b)$ and $m(b+a)$. If $a+b<b+a$, then by Lemma 3-1, $(m+1) a$ $+(m+1) b>m b+m a$, and by Lemma $2-1, \quad m b+m a \geq m(b+a)>m(a+b)$. (iii) If $b+a \geq a+b$, then by Lemma $2-1,(m+1)(a+b) \geq(m+1) a+(m+1) b$, and by Lemma $3-1,(m+1) a+(m+1) b>m a+m b$ and $m b+m a$. If $a+b$ 
$>b+a$, then by Lemma $2-1, \quad(m+1)(a+b)>(m+1)(b+a)>(m+1) b$ $+(m+1) a$, and by Lemma $3-1,(m+1) b+(m+1) a>m b+m a$ and $m a+m b$.

Remark. Lemmas 3-1 and 3-2 remain true if $P$ is an ordinal sum of pos. o.s. In fact, the given proofs apply.

For $a$ and $b$ in $P$ we define that $a \sigma b$ if $(m+1) a>m b$ and $(m+1) b>m a$ for all positive integers $m$.

1) $\sigma$ is a congruence relation. For clearly $\sigma$ is symmetric and $a \sigma a$ because $(m+1) a>m a$. If $a \sigma b$ and $b \sigma c$, then $(m+2) a>(m+1) b>m c$ and $(m+2) c$ $>(m+1) b>m a$ for all $m$. Let $m=2 n$, then $2(n+1) a>2 n c$ and $2(n+1) c$ $>2 n a$. Hence $(n+1) a>n c$ and $(n+1) c>n a$, and $a \sigma c$. Finally suppose that $a \sigma b$. By Lemma 3-2, $(m+3)(a+c)>(m+2) a+(m+2) c>(m+1) b+(m+1) c$ $>m(b+c)$ for all $m$. Let $m=3 m$, then $3(n+1)(a+c)>3 n(b+c)$. Thus $(n+1)(a+c)>n(b+c)$ and similarly $(n+1)(b+c)>n(a+c)$ for all $n$. Therefore $(a+c) \sigma(b+c)$.

2) The semigroup $P / \sigma$ is commutative. For by (i) of Lemma 3-2, $(m+1)(a+b)>m(b+a)$ and $(m+1)(b+a)>m(a+b)$ for all $m$. Therefore $(a+b)_{\sigma}(b+a)$.

For the remainder of this section we shall denote the elements of $P$ by $a, b, c$ and the elements of $P / \sigma$ by $A, B, C$. Moreover, $m, n, p, q$ will always denote positive integers. If $\rho$ is a congruence relation over a semigroup $S$, then $\rho^{*}$ will always denote the natural homomorphism of $S$ onto $S / \rho . \quad P / \sigma$ is an ordinal sum of pos. o.s., and this can be shown by verifying that $P / \sigma$ satisfies the four properties of Theorem 3-1. But we wish to show something stronger. Namely, that $P / \sigma$ is an ordinal sum of pos. o.s. each of which is a subsemigroup of positive reals.

3) If $a>b$, then $a \sigma^{*}=b \sigma^{*}$ or $x>y$ for all $x$ in $a \sigma^{*}$ and $y$ in $b \sigma^{*}$. For suppose that there exists an $x$ in $a \sigma^{*}$ and $y$ in $b \sigma^{*}$ such that $y \geq x .(m+2) x$ $>(m+1) a>(m+1) b>m y$. Now let $m=2 n$ and cancel. Then $(n+1) x>n y$ for all $n$ and also $(n+1) y \geq(n+1) x>n x$ for all $n$. Thus $x \sigma y$, and $a \sigma^{*}=x_{\sigma}^{*}$ $=y \sigma^{*}=b \sigma^{*}$. For $a \sigma^{*}$ and $b \sigma^{*}$ in $P / \sigma$ we define that $a \sigma^{*}<b \sigma^{*}$ if $a \sigma^{*} \neq b \sigma^{*}$ and $a<b$ in $P$. Then by (3) this definition is independent of the choice of representatives $a$ and $b$.

Lemma 3-3. (i) $P / \sigma$ is an ordered set and $A<B$ implies that $A+C$ 
$\leq B+C$ for all $A, B, C$ in $P / \sigma$. (ii) $A<A+A$. (iii) If $A<B$, then $n A<n B$.

Proof. (i) If $a \sigma^{*}<b \sigma^{*}$ and $b \sigma^{*}<c \sigma^{*}$, then $a<b$ and $b<c$. Hence $a<c$ and $a \sigma^{*} \leq c \sigma^{*}$. If $a \sigma^{*}=c \sigma^{*}$, then $a \in c \sigma^{*}$, but then $a>b$, a contradiction. Thus $a \sigma^{*}<b \sigma^{*}$. If $a \sigma^{*} \neq b \sigma^{*}$, then $a<b$ or $b<a$, and so $a \sigma^{*}<b \sigma^{*}$ or $b \sigma^{*}<a \sigma^{*}$. (ii) Clearly $A \leq 2 A$. Suppose that $2 A=A=a \sigma^{*}$. Then $a \sigma 2 a$, and hence $(m+1) a>(2 m) a$ for all $m$. In particular for $m=1,2 a>2 a$, a contradiction. Thus $A<A+A$. (iii) Clearly $n A \leq n B$. Suppose that $n A=n B$ where $a \sigma^{*}=A$ and $b \sigma^{*}=B$. Then $n a \sigma n b$, and so $(m+1) n a>m n b$ and $(m+1) n b>m n a$ for all $m$. But then $(m+1) a>m b$ and $(m+1) b>m a$. Thus $a \sigma b$, and hence $A=a \sigma^{*}=b \sigma^{*}=B$, a contradiction.

For $A$ and $B$ in $P / \sigma$ we define that $A \tau B$ if there exist positive integers $m$ and $n$ such that $m A>B$ and $n B>A$.

4) $\tau$ is an equivalence relation. For clearly $r$ is symmetric and by (ii) of Lemma $3-3,2 A>A$. Thus $A \tau A$. If $A \tau B$ and $B \tau C$, then $n A>B, p B>A$, $m B>C$ and $q C>B$ for some positive integers $m, n, p, q$. By (iii) of Lemma 3-3, $m n A>m B>C$ and $p q C>p B>A$. Therefore $A \tau C$.

Let $A \tau^{*}$ be the equivalence class that contains $A$. We shall show later that $\tau$ is a congruence relation, and so $\tau^{*}$ is the natural homomorphism of $P / \sigma$ onto $(P / \sigma) / \tau$.

5) If $A<B$ and $A \tau^{*} \neq B \tau^{*}$, then $A \tau^{*}<B \tau^{*}$ and $A+B=B$. For suppose that there exist $X$ in $A \tau^{*}$ and $Y$ in $B \tau^{*}$ such that $X \geq Y$. Then $n X \geq n Y>B$ and $m B \geq m A>X$ for some $m$ and $n$. Thus $X \tau B$, and hence $A \tau^{*}=X \tau^{*}=B \tau^{*}$. $A=a \sigma^{*}$ and $B=b \sigma^{*}$. Since $a+b>b,(m+1)(a+b)>m(a+b)>m b$ for all $m$. Thus it suffices to show that $(m+1) b>m(a+b)$ for all $m$. Now $n A<B$ for all $n$, for otherwise $A \in B \tau^{*}$. Thus $n a<b$ for all $n$. $(n+2) b=b+(n+1) b$ $>(n+1) a+(n+1) b>n(a+b)$. Now let $n=2 m$ and cancel to get $(m+1) b$ $>m(a+b)$. Thus $A+B=B$.

6) If $A<B$ and $A \tau C$, then $A+C<B+C$. For $A=a \sigma^{*}, B=b \sigma^{*}, C=c \sigma^{*}$, $a<b$ and $(n+1) a<n b$ for some positive integer $n$. By Lemma 3-3, $A+C$ $\leq B+C$. Suppose (by way of contradiction) that $A+C=B+C$. Then $(m+3) a$ $+(m+3) c>(m+2)(a+c)>(m+1)(b+c)>m b+m c$. Therefore $(m+3) a$ $+3 c>m b$ for all $m$. Since $A \tau C$, there exists an integer $h$ such that $h A$ $>C$ and $3 h A>3 C$ by Lemma .3-3. Let $k=3 h$, then $k a>3 c .(k+3) n b$ 
$>(k+3)(n+1) a=[(k+3) n+3] a+k a>[(k+3) n+3] a+3 c$. Now let $m=(k+3) n$. Then $m b>(m+3) a+c$, a contradiction.

TheOREM 3-2. For each $A$ in $P / \sigma, A \tau^{*}$ is an ordered subsemigroup of $P / \sigma$ that is o-isomorphic to an additive semigroup of positive real numbers. $P / \sigma$ is an ordinal sum of the pos. o.s. $A \tau^{*}$.

Proof. Consider $B, C$ in $A \tau^{*} . A=a \sigma^{*}, B=b \sigma^{*}$ and $C=c \sigma^{*}$, where $a, b, c$ $\in P$. There exist positive integers $m, n, r, s$ such that $m B>A, n A>B, r C>A$ and $s A>C$. Thus $m b>a, n a>b, r c>a$ and $s a>c$. Let $q=\max \{m, r\}$. Then $q b>a$ and $q c>a$. Thus $(q+1)(b+c)>q b+q c>2 a>a$, and by Lemma $3-3,(q+2)(B+C)>(q+1)(B+C) \geq A$. Let $t=\max \{n, s\}$. Then $t a>b$ and $t a>c$. Thus $2 t a>b+c$ and $(2 t+1) A>2 t A \geq B+C$. Therefore $B+C \in A \tau^{*}$, and so $A \tau^{*}$ is a semigroup. By Lemma $3-3, A \tau^{*}$ is ordered, and thus by (6) $A \tau^{*}$ is an o.s. In order to prove that $A^{*}$ is $o$-isomorphic to a semigroup of positive real numbers, it suffices by Theorem 2-3 to show that if $X, Y \in A \tau^{*}$ and $X<Y$, then there exist positive integers $m$ and $n$ such that $(m+1) X<m Y$ and $n X<(n+1) Y$.

$X=x \sigma^{*}$ and $Y=y \sigma^{*}$ for some $x$ and $y$ in $\mathrm{P}$. Since $X<Y, n X<n Y$ $<(n+1) Y$ for all $n$. Hence $n x<(n+1) y$ for all $n$. Suppose (by way of contradiction) that $(m+1) X \geq m Y$ for all $m$. If for some $m,(m+1) X=m Y$, then $(m+2) X=(m+1) X+X<m Y+Y=(m+1) Y$. Therefore $(m+1) X$ $>m Y$ for all $m$. Thus $(m+1) x>m y$ and $m x<(m+1) y$ for all $m$. Therefore $X=Y$, a contradiction. Thus by Theorem $2-3$ there exists an isomorphism $\pi$ of $A \tau^{*}$ into the additive group of reals. But for $B \in A \tau^{*}, B<2 B$. Hence $B \pi$ $<2(B \pi)$. Therefore $B \pi$ is a positive real number. It follows at once from (4) and (5) that $P / \sigma$ is the ordinal sum of the $A \tau^{*}$.

COROLlary. $\tau$ is a congruence relation on $P / \sigma$.

Proof. Consider $X, Y, Z$ in $P / \sigma$, and assume that $X \tau Y$. If $Z \tau X$, then since $X \tau^{*}$ is a semigroup $X+Z$ and $Y+Z$ belong to $X \tau^{*}$. Thus $(X+Z) \tau(Y+Z)$. Suppose that $X \tau^{*} \neq Z \tau^{*}$. If $Z<X$, then $Z \tau^{*}<X \tau^{*}=Y \tau^{*}$. Thus by (5) $X+Z$ $=X$ and $Y+Z=Y$. If $X<Z$, then $Y \tau^{*}=X \tau^{*}<Z \tau^{*}$. Thus by (5) $X+Z=Z$ $=Y+Z$. In either case $(X+Z) \tau(Y+Z)$.

There is a natural 1-1 order preserving correspondence between the congruence relations of $P / \sigma$ and the congruence relations of $P$ that contain $\delta$, 
Therefore $\tau$ can also be considered as a congruence relation on $P$, where $a \tau b$ if there exist positive integers $m$ and $n$ such that $m a>b$ and $n b>a$. Consider $X$ and $Y$ in $P / \tau . \quad X=x \tau^{*}$ and $Y=y \tau^{*}$ for some $x$ and $y$ in $P$. We define that $X<Y$ if $X \neq Y$ and $x<y$ in $P$. Then $P / \tau$ is an ordered set and $\tau^{*}$ is an $o$ homomorphism of $P$ onto $P / \tau$. Denote the addition in $P / \tau$ by $[+]$. Then since $X+Y \subseteq \max [X, Y]$ in $P, X[+] Y=\max [X, Y]$ in $P / \tau . \quad X$ is a subsemigroup of $P$ and $X / \sigma$ is $o$-isomorphic to a subsemigroup of the positive reals. Thus in Clifford's terminology [3], $P / \tau$ is a semilattice and $P$ is a semilattice of the semigroups $X \in P / \tau$. In particular, $P-X$ is a subsemigroup of $P$ and the number of components $A \sigma^{*}$ of $P / \sigma$ is equal to the number of elements in $P / \tau$ which we shall denote by $|P / \tau|$.

A subsemigroup $C$ of $P$ is convex if $a \subseteq P, c \in C$ and $a<c$ imply that $a \in C$. It is easy to show that the set $\mathscr{S}$ of all convex subsemigroups of $P$ is ordered by inclusion, and that if $A$ and $B$ are convex subsemigroups of $P$ and $A \supset B$, then $A \backslash B$ is a semigroup. Moreover if $A$ covers $B$, and $a \in A \backslash B$, then $a \tau^{*}=A \backslash B$. For each $a \in P$ let $P^{a}=\left\{x \in P: x \tau^{*} \leq a \tau^{*}\right\}$. Then $P^{a}$ is a convex subsemigroup of $P$ and if $C$ is a convex subsemigroup of $P$, then $C=\bigcup_{a \in C} P^{a}$. Thus the order type of $\mathscr{S}$ is completely determined by $P / \tau$.

Let $G$ be an $o$-group and let $\Gamma$ be the set of all pairs of convex subgroups $G^{\gamma}, G_{\Upsilon}$ of $G$ such that $G^{*}$ covers $G_{r}$. Define that $\left(G_{\alpha}, G^{\alpha}\right)<\left(G_{\beta}, G^{3}\right)$ if $G^{\alpha} \leq G_{\beta}$. Then $\Gamma$ is ordered, and the order type of $\Gamma$ is the rank of $G$.

THEOREM 3-3. If $P$ is a naturally pos. o.s., then the rank of the difference grout $G$ of $P$ equals the order type of $P / \tau$.

For by Theorem 2-2, $P$ is the semigroup of all positive elements of $G$, and a convex subgroup of $G$ is determined by its set of positive elements. Thus if $\left(G_{r}, G^{r}\right) \in I$, then $G_{r} \cap P$ and $G^{r} \cap P$ are convex subsemigroups of $P$ and $G^{\Upsilon} \cap P$ covers $G_{;} \cap P$. Moreover $\left(G^{\Upsilon} \cap P\right) \backslash\left(G_{r} \cap P\right)=a \tau^{*}$, where $a \in\left(G^{\Upsilon} \cap P\right)$ $\backslash(G \backsim \cap)$.

Remark. If $P$ is a commutative naturally pos. o.s. and the components $A \tau^{*}$ of $P / \sigma$ are $d$-closed, then the $c$-closure $C$ of the difference group $G$ of $P$ is uniquely determined by $P / \sigma$. For $C$ is isomorphic to the Hahn group $H\left(T, R_{r}\right)$, where $I$ is an ordered set with order type equal to the rank of $G$ and the $R_{-}$ are isomorphic to the components $G^{\check{r}} / G_{r}$ of $G$ (see [5] for these concepts). But $\Gamma$ is determined by $P / \sigma$ and the components of $G$ are just the difference 
groups of the components of $P / \sigma$.

Let $P$ be a positive o.s. that satisfies $(*)$ and let $G$ be the difference group of $P$. It should be made clear that there is virtually no relationship between the order type of $P / \tau$ and the rank of $G$, even if $G$ is abelian. For example let $G=R \oplus R \oplus R$, where $R$ is the additive group of real numbers. Define $(a, b, c)$ in $G$ positive if $c>0$ or $c=0$ and $b>0$ or $c=b=0$ and $a>0$. Let $P=\{(a, b, c)$ $\in G: c>0\}$. Then $G$ is the difference group of $P,|P / \tau|=1$, and the rank of $G$ is 3. By generalizing this example it is easy to see that for $|P / \tau|=1$ the rank of $G$ can be any given order type. But we shall show (Theorem 5-1) that $P$ does determine the order type of the set of all convex normai subgroups of $G$.

4. Relationships between $P$ and $P / \sigma$. Throughout this section let $P$ be a pos. o.s. A semigroup $Q$ is a $t$-semigroup if $Q$ is an ordered set and $m a<(m+1) a$ for all $a$ in $Q$ and all positive integers $m$.

LeMma 4-1. Let $\rho^{*}$ be an o-homomorphism of Ponto a $t$-semigroup $Q$. For $a$ and $b$ in $P$ define $a \rho b$ if $a \rho^{*}=b \rho^{*}$. Then $\rho$ is a congruence relation on $P$ and $\rho \subseteq \sigma$.

Proof. If $a \rho b$, then $a \rho^{*}=b \rho^{*}$. Hence $(m+1)\left(a \rho^{*}\right)>m\left(b \rho^{*}\right)$ and $(m+1) \cdot$ $\left(b \rho^{*}\right)>m\left(a \rho^{*}\right)$. Thus since $\rho^{*}$ is an $o$-homomorphism, $(m+1) a>m b$ and $(m+1) b>m a$ for all $m$. Therefore $a \sigma b$.

Now consider $q \in Q . \quad q=a \rho^{*}$ for some $a \in P$. Define $q \alpha=a \sigma^{*}$. Then by the usual arguments $\alpha$ is an $o$-homomorphism of $Q$ onto $P / \sigma$ such that $p \rho^{*} \alpha$ $=p \sigma^{*}$ for all $p \in P$. We have the following diagram and theorem.

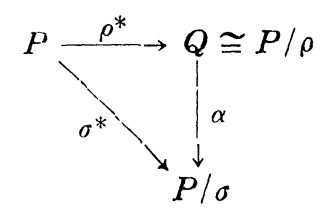

Theorem 4-1. $P / \sigma$ is the smallest o-homomorphic image of $P$ that is a $t$ semigroup. In particular, $P / \sigma$ is the smallest o-homomorphic inage of $P$ that is an ordinal sum of pos. o.s.

Remarks. (1) Let $\rho$ be a congruence relation on $P$. Then $P / \rho$ is a $t$ semigroup and $\rho^{*}$ is an $o$-homomorphism if and only if for all $a, b \in P:(A)$ If $a<b$, then $a \rho^{*}=b \rho^{*}$ or $x<y$ for all $x \in a \rho^{*}$ and $y \in b \rho^{*}$, and $m a$ (NOT $\rho$ ) 
$(m+1) a$ for all $m$. Thus $\sigma$ is the join of all congruence relations that satisfy (A). (2) If $|P / \tau|=1$ and $\rho$ is a congruence relation on $P$ such that $P / \rho$ is an ordinal sum of pos. o.s. and $\rho^{*}$ is an $o$-homomorphism, then $P / \rho$ is a pos. o.s..

5. Relationship between $P$ and its quotient group $G$. Let $P$ be a pos. o.s. and let $\Delta=\{\rho: \rho$ is a congruence relation on $P, P / \rho$ is a pos. o.s. or a pos. o.s. with zero, and $\rho^{*}$ is an $o$-homomorphism $\}$.

LeMma 5-1. $\Delta$ is ordered by inclusion.

Proof. Consider $\alpha, \beta \in \Delta$ and suppose (by way of contradiction) that there exist $a, b, c, d \in P$ such that $a \alpha b, a$ (NOT $\beta$ ) $b, c$ (NOT $\alpha) d$ and $c \beta d$. Case I. $a>b$ and $c>d$. Then $a \beta^{*}>b \beta^{*}$ and $c \alpha^{*}>d \alpha^{*}$. If $a+d \leq b+c$, then $a \beta^{*}+d \beta^{*}$ $\leq b \beta^{*}+c \beta^{*}$ and $d \beta^{*}=c \beta^{*}$. Thus $a \beta^{*} \leq b \beta^{*}$, a contradiction. If $a+d>b+c$, then $a \alpha^{*}+d \alpha^{*} \geq b \alpha^{*}+c \alpha^{*}$ and $a \alpha^{*}=b \alpha^{*}$. Thus $d \alpha^{*} \geq c \alpha^{*}$, a contradiction. Similarly in the other three cases we get a contradiction.

For the remainder of this section we assume that $P$ is a pos. o.s. which satisfies $(*)$. In particular, the results obtained are valid for commutative pos. o.s. Let $G$ be the difference group of $P$ and let $\pi$ be an $o$-homomorphism of $P$ into a pos. o.s. with zero. Then clearly $P_{\pi}$ satisfies (*). Let $H$ be the difference group of $P \pi$ and for $g=a-b$ in $G$ define $g \bar{\pi}=a \pi-b \pi$.

Lemma $5-2 . \quad \bar{\pi}$ is the unique extension of $\pi$ to an o-homomorphism of $G$ onto $H$.

Proof. If $a-b=c-d$, where $a, b, c, d \in P$, then by Corollary II of Theorem $2-1$, there exist $x, y \in P$ such that $a+x=c+y$ and $b+x=d+y$. Thus $a \pi+x \pi$ $=c \pi+y \pi$ and $b \pi+x \pi=d \pi+y \pi$, and so by applying this corollary again, $a \pi-b \pi$ $=c \pi-d \pi$. Thus $\bar{\pi}$ is single valued. The lemma now follows by repeated use of Corollary II and straightforward computation.

It is well known and easy to verify that the kernel of any $o$-homomorphism of an o-group is a convex normal subgroup. Let $\mathscr{C}$ be the set of all convex normal subgroups of $G$ except $G$ itself. Then $\mathscr{C}$ is ordered with respect to inclusion.

TheOREM 5-1. There exists a 1-1 order preserving mapping of 4 onto $\mathscr{C}$.

Proof. For each $\rho \in \Delta$ let $\bar{\rho}$ be the unique extension of $\rho^{*}$ to $G$ (which is assured by Lemma $5-2$ ), and let $\rho \eta=K(\bar{\rho})=\{x \in G: x \bar{\rho}=0\}$. We wish to show 
that $\eta$ is the desired mapping. Since $\bar{\rho}$ is uniquely determined by $\rho, \eta$ is single valued. Let $\alpha, \beta \in \Delta$ and $\alpha \subseteq \beta$. If $x \in K(\bar{\alpha})$, then $x=a-b$, where $a, b \in P$ and $0=x \bar{\alpha}=(a-b) \bar{\alpha}=a \bar{\alpha}-b \bar{\alpha}=a \alpha^{*}-b \alpha^{*}$. Thus $a \alpha b$ and hence $a \beta b$. But then $0=a \beta^{*}-b \beta^{*}=x \dot{\beta}$. Therefore $x \in K(\bar{\rho})$ and $\alpha \eta \subseteq \beta \eta$. If $\alpha \neq \beta$, then there exist $a, b \in P$ such that $a \beta b$ but not $a \alpha b$, but this means that $a-b \in K(\bar{\beta}) \backslash K(\bar{\alpha})$. Therefore $\eta$ is $1-1$ and order preserving. Next consider $C \in \mathscr{C}$ and let $N$ be the natural $o$-homomorphism of $G$ onto $G / C$. Let $\rho$ be the congruence relation induced on $P$ by $N(a \rho b$ if and only if $a N=b N)$. Define that $a \rho^{*}>b \rho^{*}$ if $a+C$ $>b+c$. Then it follows by a straightforward computation that $\rho \in \Delta$ and $\rho \eta=C$. Therefore $\eta$ is $a 1-1$ orderpreserving mapping of $\Delta$ onto $\mathscr{C}$.

If $|P / \tau|=1$ or equivalently if $P / \sigma$ is $o$-isomorphic to a subsemigroup of positive reals, then $\sigma \in \Delta$ and $\Delta=\{\rho: \rho$ is a congruence relation on $P, P / \rho$ is a pos. o.s. and $\rho^{*}$ is an $o$-homomorphism $\}$.

\section{REFERENCES}

[1] N. G. Alimov, On ordered semigroups, Izv. Akad. Nauk SSSR Ser. Mat. 14 (1950), $569-576$.

[2] C. G. Chehata, On ordered semigroups, J. London Math. Soc. 28 (1953), 353-356.

[3] A. H. Clifford, Bands of semigroups, Proc. Amer. Math. Soc. 5 (1954), 499-504.

[4] A. H. Clifford, Totally ordered commutative semigroups, Bull. Amer. Math. Soc. 64 (1958), 305-316.

[5] P. Conrad, Extensions of ordered groups, Proc. Amer. Math. Soc. 6 (1955), 516-528.

[6] P. Conrad, Semigroups of real numbers, (to appear).

[7] C. J. Everett, A note on a result of L. Fuchs on ordered groups, Amer. J. Math. 73 (1950), 216.

[8] O. Ore, Linear equations in non-commutative fields, Annals Math. 32 (1931), 463-477.

[9] A. A. Vinogradov, On the theory of ordered semigroups, Ivanov. Gos. Ped. Inst. Uc. Zap. Fiz. Mat. Nauki 4 (1953) 19-21.

\section{Tulane University}

